\title{
Cor Triatriatum Associated with Total Anomalous Pulmonary Venous Connection: A Rare but Plausible Combination
}

\author{
Jun Oh Lee, M.D., Chun Soo Park, M.D., Ph.D. \\ Department of Thoracic and Cardiovascular Surgery, Asan Medical Center, University of Ulsan College of Medicine, Seoul, Korea
}

\section{ARTICLE INFO}

Received May 8, 2020

Revised July 10, 2020

Accepted July 11, 2020

Corresponding author

Chun Soo Park

Tel $82-2-3010-3583$

Fax 82-2-3010-6966

E-mail chunsoo@amc.seoul.kr;

hopang1974@hanmail.net

ORCID

https://orcid.org/0000-0001-8718-8904

\begin{abstract}
In a newborn in whom cor triatriatum was missed on echocardiography, infracardiac total anomalous pulmonary venous connection was successfully repaired with the aid of cardiac computed tomography (CT). In rare combinations, as in this case, an accurate diagnosis prior to surgery, which is of vital importance for successful repair, can be made through a high index of suspicion and the use of a supplemental imaging modality such as CT.
\end{abstract}

Keywords: Congenital heart disease, Cor triatriatum, Total anomalous pulmonary venous return

\section{Case report}

Although the association of total anomalous pulmonary venous connection (TAPVC) with cor triatriatum seems implausible, this rare combination has been convincingly documented and embryologically rational explanations have been proposed $[1,2]$. The rarity of this combination means that cardiologists or cardiac surgeons might not clearly identify and elaborate the anatomic details before surgery unless they have a high index of suspicion. However, complementary to echocardiography, additional imaging modalities such as cardiac computed tomography (CT) can provide critical information for making an accurate diagnosis. Here, we report the case of a baby born with this rare combination that was successfully repaired, and we present 1-year follow-up imaging.

An 8-day-old newborn was transferred to Asan Medical Center with severe respiratory distress and hypoxia requiring immediate mechanical ventilatory support. Transthoracic echocardiography (TTE) demonstrated TAPVC, which drained into the portal vein through the vertical vein without flow disturbance. A common pulmonary venous chamber accommodating all the pulmonary venous blood was identified, but it was not thought based on TTE that a part of the common pulmonary venous chamber was the proximal chamber of the left atrium (LA).

A cardiac CT scan clearly demonstrated infracardiac TAPVC, which was compatible with the TTE findings. In contrast to TTE, the CT scan also revealed a round chamber communicating with all the pulmonary veins that mimicked the LA, which had collapsed (Fig. 1). This finding suggested an obstructed cor triatriatum associated with infracardiac TAPVC. The descending vertical vein drained into the portal vein and there was no evidence of stenosis or narrowing.

At 12 days of age, the patient underwent urgent repair under moderate hypothermic cardiopulmonary bypass. An incision was made in the midline of the sternum and perfusion cannulas were inserted through the aorta, right atrial appendage, and inferior vena cava. After commencing cardiopulmonary bypass, 4 individual pulmonary veins, a posteriorly located pulmonary venous confluence, and the descending vertical vein were clearly identified. Under cardioplegic arrest, the right atrium opened and a thick muscular membrane was seen posteriorly through the widely opened atrial septal defect (ASD). The membrane was incised at the center and widely resected to establish free communication between the proximal and distal (accessory and true) LA chambers. To enhance pulmonary venous inflow, an incision on the posterior wall of the proximal LA 

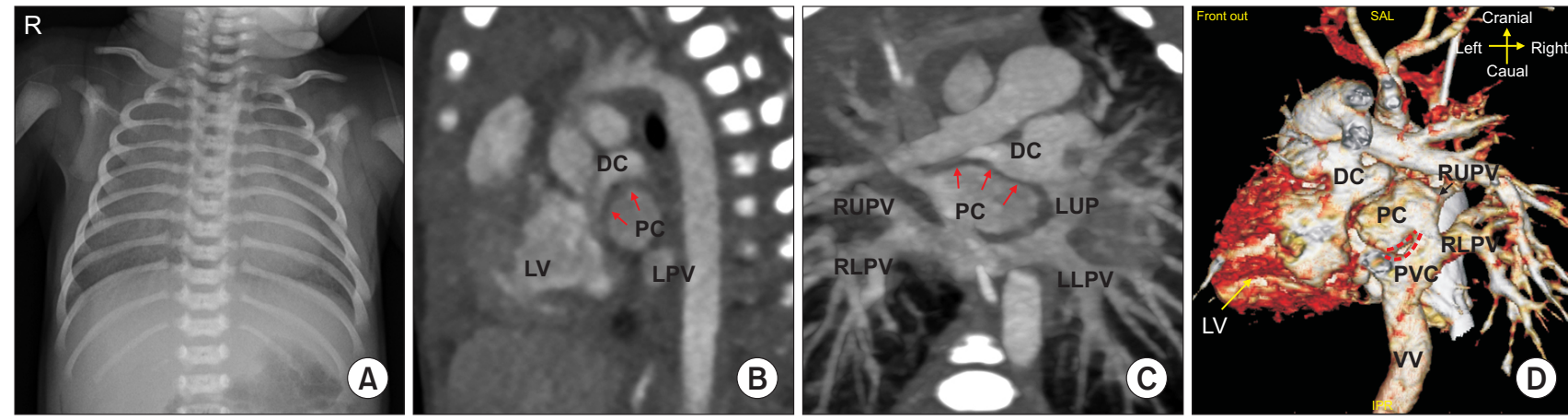

Fig. 1. (A) A preoperative simple chest X-ray shows multifocal dependent opacities in both lungs due to pulmonary congestion. (B, C) The thick membrane between the 2 left atrial (A) chambers (arrow) bulged toward the anteriorly positioned collapsed true left atrium (DC) due to cor triatriatum (PC) in (B) sagittal and (C) coronal section CT images. (D) In the volume-rendered CT image, the extent of incisions of the PVC and proximal left atrium can be delineated (red dotted line). DC, distal chamber; PC, proximal chamber; CT, computed tomography; LV, left ventricle; LPV, left pulmonary vein; LLPV, left lower pulmonary vein; RLPV, right lower pulmonary vein; RUPV, right upper pulmonary vein; LUPV, left upper pulmonary vein; PVC, pulmonary venous confluence; VV, vertical vein.
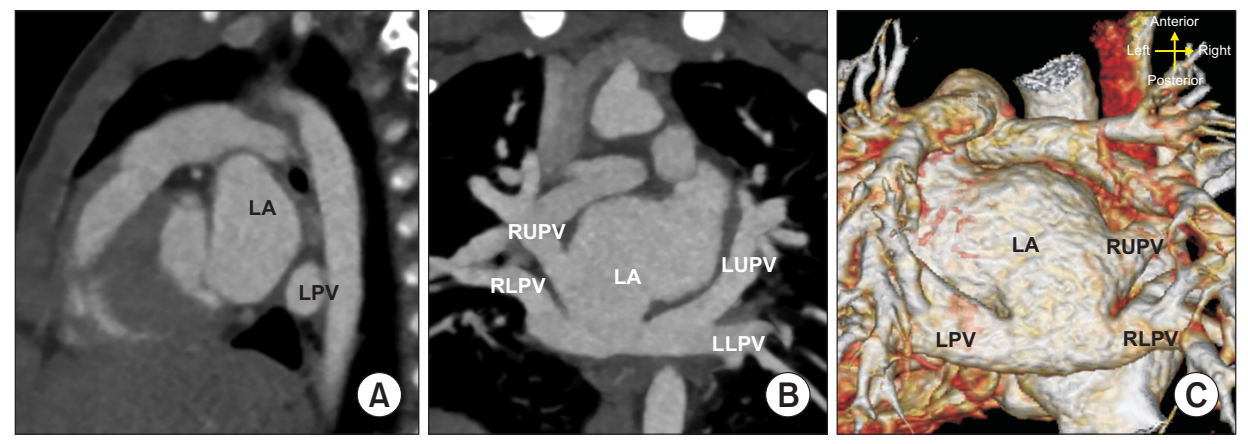

Fig. 2. One-year follow-up computed tomography scan. (A) No residual membranous structures were identified in the left atrial chamber. (B, C) Individual pulmonary veins and the junction between the pulmonary venous confluence and LA were unobstructed in (B) the coronal section and (C) volume-rendered images. LA, left atrium; LPV, left pulmonary vein; LLPV, left lower pulmonary vein; RLPV, right lower pulmonary vein; RUPV, right upper pulmonary vein; LUPV, left upper pulmonary vein.

chamber and a corresponding incision on the anterior wall of the pulmonary venous confluence were anastomosed with 8-0 Prolene sutures in an interlocked continuous running fashion. The ASD was closed with bovine pericardium. The right atrial incision was repaired, and the descending vertical vein was divided. Convalescence from cardiopulmonary bypass was uneventful, and the patient was transferred to the pediatric intensive care unit with the sternum remaining open. The sternum was closed on postoperative day 2. The patient was taken off of mechanical ventilator support on postoperative day 13 , transferred to the general ward on postoperative day 15 , and discharged on postoperative day 31 .

Follow-up TTE at discharge revealed no individual pulmonary vein stenosis, no flow disturbance in the LA, no evidence of pulmonary hypertension, and normal ventricular function. At the 1-year follow-up, the patient was do- ing well without medication, an electrocardiogram was unremarkable, and unobstructed individual pulmonary veins and LA were demonstrated on a CT scan (Fig. 2).

The patient's caregiver provided informed consent for publication of this research including medical records and clinical images on the line.

\section{Discussion}

Cor triatriatum is a rare congenital cardiac defect accounting for $0.1 \%-0.4 \%$ of all cases of congenital heart disease. In cor triatriatum, the LA is divided into 2 chambers by an abnormal membranous structure, which can hinder the inflow toward the left ventricle (LV) and consequently function as a pulmonary venous obstruction resulting in pulmonary hypertension and cardiac failure. TAPVC is a well-known pulmonary venous anomaly that is also fre- 
quently accompanied by a pulmonary venous obstruction. Therefore, without adequate surgical treatment, concurrent existence of both anomalies could lead to severe morbidity and even death despite its extremely rare incidence $[3,4]$. An accurate diagnosis prior to surgery is paramount for successful repair, but the co-existence of these 2 abnormalities is barely detectable using traditional TTE unless the physician has a high index of suspicion. In this case, the presence of associated cor triatriatum could not be identified by TTE, but was finally detected on a CT scan, whereby the anatomical relationship between the individual pulmonary veins and proximal and distal chambers of the LA were clearly delineated.

The classification of cor triatriatum was first suggested by Loeffler [5] and subsequently elaborated by Niwayama [6], Grondin et al. [7], and Krabill and Lucas [8]. According to the classification of Lucas, our case was compatible with cor triatriatum type $\mathrm{B} 2$, where the accessory chamber receives all pulmonary veins without communication between the accessory and true LA chambers, and pulmonary venous blood drains into the systemic vein in the fashion of TAPVC [1].

Patients with cor triatriatum associated with TAPVC can present with low cardiac output and cardiogenic shock due to insufficient LV preload resulting from the limited size of the interchamber (proximal and distal LA) and interatrial communication, which is the sole channel toward the LV. As medical treatment usually does not work well for patients with mechanically obstructed pulmonary venous inflow, timely surgical repair is crucial for a favorable outcome. Although surgery was delayed to ensure an accurate diagnosis, our patient was stably maintained with a low inotrope dosage and minimum respiratory support until repair, owing to the grossly unobstructed pulmonary venous drainage. The surgical procedures are quite straightforward: the membrane should be resected as much as possible and anomalously returned pulmonary veins should be widely connected to the LA. In this case, the membrane was widely resected, following standard practice, and TAPVC was repaired in a manner similar to conventional repair, with the proximal left atrial chamber and pulmonary venous confluence anastomosed from the pulmonary venous opening in the proximal LA.

Pulmonary vein stenosis is the most frequent and fatal complication after TAPVC repair. Most cases of pulmonary vein stenosis occur within 1 year following TAPVC repair. Fortunately, the 1-year follow up image in our case showed no evidence of pulmonary venous inflow obstruction.

In conclusion, an accurate diagnosis prior to surgery, which is of vital importance for successful repair, can be established through a high index of suspicion and the use of a supplemental imaging modality such as CT for this rare combination of cor triatriatum and TAPVC. An accurate diagnosis prior to surgery can enable satisfactory outcomes to be achieved despite delayed referral and repair.

\section{Conflict of interest}

No potential conflict of interest relevant to this article was reported.

\section{ORCID}

Jun Oh Lee: https://orcid.org/0000-0003-3373-1144

Chun Soo Park: https://orcid.org/0000-0001-8718-8904

\section{References}

1. Herlong JR, Jaggers JJ, Ungerleider RM. Congenital Heart Surgery Nomenclature and Database Project: pulmonary venous anomalies. Ann Thorac Surg 2000;69(4 Suppl):S56-69.

2. Man JB, Chan YN, Sam SO, et al. Cor triatriatum with infracardiac total anomalous pulmonary venous drainage. Korean J Thorac Cardiovasc Surg 2002;35:52-5.

3. Burroughs JT, Edwards JE. Total anomalous pulmonary venous connection. Am Heart J 1960;59:913-31.

4. Vouhe PR, Baillot-Vernant F, Fermont L, Bical O, Leca F, Neveux JY. Cor triatriatum and total anomalous pulmonary venous connection: a rare, surgically correctable anomaly. J Thorac Cardiovasc Surg 1985;90:443-5.

5. Loeffler E. Unusual malformation of the left atrium: pulmonary sinus. Arch Pathol (Chic) 1949;48:371-6.

6. Niwayama G. Cor triatriatum. Am Heart J 1960;59:291-317.

7. Grondin C, Leonard AS, Anderson RC, Amplatz KA, Edwards JE, Varco RL. Cor triatriatum: a diagnostic surgical enigma. J Thorac Cardiovasc Surg 1964;48:527-39.

8. Krabill KA, Lucas RV. Abnormal pulmonary venous connections. In: Emmanoulides GC, Riemenschneider TA, Allen HD, Gutgesselle, editors. Heart disease in infants, children, and adolescents: including the fetus and young adult. Baltimore (MD): Williams and Wilkins; 1995. p. 838-74. 